\title{
The 2021 European Heart Failure Guidelines: The Case for Personalised Therapeutics
}

\author{
Nesan Shanmugam (1) \\ St George's University Hospitals NHS Foundation Trust, St George's University of London Cardiology Clinical Academic Group, London, UK
}

\section{Keywords}

Heart failure, guidelines, prevention, patient-centred care

Disclosure: NS is on the European Cardiology Review editorial board.

Received: 22 November 2021 Accepted: 23 November 2021 Citation: European Cardiology Review 2022;17:e01. D0I: https://doi.org/10.15420/ecr.2021.57

Correspondence: Nesan Shanmugam, St George's University Hospitals NHS Foundation Trust, Blackshaw Rd, Tooting, London SW17 0QT, UK.

E: Nesan.shanmugam@stgeorges.nhs.uk

Open Access: This work is open access under the CC-BY-NC 4.0 License which allows users to copy, redistribute and make derivative works for non-commercial purposes, provided the original work is cited correctly.

The field of heart failure (HF) has seen the development of unparalleled evidence-based therapies over the last 30 years, with continuous improvement in survival and quality of life for our patients. It is unquestionably an exciting time to be considering or pursuing HF as a subspecialty. Pivotal to and underpinning these advances is the guidance provided by international HF guidelines, particularly from the European Society of Cardiology (ESC). The new 2021 ESC HF guidelines have a total of 41 new and 15 modified recommendations from the 2016 document.

A significant feature of the new guidelines is the focus on patient-centred care. Notably, this is the first ESC guideline to include patients as full members of the task force, with patients at the centre of the management algorithm in partnership with the multidisciplinary team.

There has been an innovative expansion in the therapeutic tool kit available for physicians managing patients with $\mathrm{HF}$ with reduced ejection fraction (HFrEF), with the focus now moving from three to four foundational classes of drugs: angiotensin-converting enzyme inhibitors (ACEI)/ angiotensin receptor-neprilysin inhibitors (ARNI), $\beta$-blockers, mineralocorticoid receptor antagonists and the new sodium-glucose cotransporter 2 (SGLT2) inhibitors. All have additive and independent treatment benefits.

It has been fascinating to see the transition of SGLT2 inhibitors from the diabetes arena to the HF world. Dapagliflozin and empagliflozin have both shown clinically significant reductions in mortality and HF hospitalisations and - importantly - improvement in quality of life when combined with gold standard triple neurohormonal modulation/blockade therapies for patients with HFrEF with or without diabetes. ${ }^{2,3}$ Known as the 'fantastic four', there is a real focus on the accelerated initiation of these powerful therapies at lower doses over a 2-week to 4-week window, rather than the traditional sequential sequencing and uptitration of the individual drug classes to target doses. ${ }^{4}$ Recent trials have clearly demonstrated the rapid positive additive treatment effects of SGLT2 inhibitors, with outcome curves diverging within the first month.
A further new recommendation is the consideration of initiation of sacubitril/valsartan in ACEI-naiive patients hospitalised with HFrEF (class $\mathrm{Ilb}$, level B). Initiation in this setting appears to be safe and dramatically reduces subsequent cardiovascular death or HF hospitalisations by $42 \%$ compared with enalapril. ${ }^{5}$

The syndrome of HF is a consequence of complex pathophysiological interactions and multiple comorbidities, and although a generic 'all sizes fits all' approach is often recommended in therapeutic guidelines; the ESC HF Task Force must be commended on their efforts to simplify and provide a phenotypic breakdown of various HF conditions. An array of nuanced therapeutic options are recommended depending on the specific patient phenotype (e.g. left bundle branch block and CRT, aortic stenosis and transcatheter aortic valve implantation). This strategic personalised approach will invariably have a positive impact on the overall prognosis of our patients.

In addition, there is a real paradigm shift to provide a more tailored, patientspecific approach using patient profiles, such as heart rate, the presence of AF, symptomatic low blood pressure, kidney function or hyperkalaemia, as guides to initiating and adjusting guideline-directed medical therapy. ${ }^{6}$ Appropriate sequencing may also enhance the tolerability of medications started later in the sequence, moving away from the previous model of rigid titration of each drug class before commencing the next treatment.

Despite these innovative evidenced-based therapies, it is also clear that delivery of guidelines varies between settings, contributing to disparities in care and worse outcomes for our patients. For example, observed use of ARNI in the UK is almost $50 \%$ below expected in primary and secondary care settings. ${ }^{7}$ This problem has been further compounded by the COVID-19 pandemic, which has led to an unprecedented restructuring of HF service provision with significant disruption to standard pathways for medication delivery. Thirty-seven per cent of patients reported disruption to medication prescription services in the UK during the pandemic. ${ }^{8}$ Therefore, there is an urgent need to redress this situation and shift back to a patient-centred approach - a central tenet of the updated 2021 ESC HF Guidelines. 
1. McDonagh TA, Metra M, Adamo M, et al. 2021 ESC guidelines for the diagnosis and treatment of acute and chronic heart failure. Eur Heart J 2021;42:3599-726. https:// doi.org/10.1093/eurheartj/ehab368; PMID: 34447992.

2. McMurray JJV, Solomon SD, Inzucchi SE, et al. Dapagliflozin in patients with heart failure and reduced ejection fraction. N Engl J Med 2019;381:1995-2008. https://doi.org/10.1056/ NEJMoa1911303; PMID: 31535829

3. Packer M, Anker SD, Butler J, et al. Cardiovascular and renal outcomes with empagliflozin in heart failure. $N$ Engl $\mathrm{J}$ Med 2020;383:1413-24. https://doi.org/10.1056/NEJMoa2022190; PMID: 32865377.

4. Straw S, McGinlay M, Witte KK. Four pillars of heart failure: contemporary pharmacological therapy for heart failure with reduced ejection fraction. Open Heart 2021;8:e001585. https://doi.org/10.1136/openhrt-2021-001585;

PMID: 33653703

5. Velazquez EJ, Morrow DA, DeVore AD, et al. Angiotensinneprilysin inhibition in acute decompensated heart failure. N Engl J Med 2019;380:539-48. https://doi.org/10.1056/ NEJMoa1812851; PMID: 30415601

6. Rosano GMC, Moura B, Metra M, et al. Patient profiling in heart failure for tailoring medical therapy. A consensus document of the Heart Failure Association of the European Society of Cardiology. Eur J Heart Fail 2021;23:872-81. https://doi.org/10.1002/ejhf.2206; PMID: 33932268.
7. NHS Digital. NICE Technology Appraisals in the NHS in England (Innovation Scorecard) to March 2020. Leeds: NHS Digital, 2020. https://digital.nhs.uk/data-and-information/ publications/statistical/nice-technology-appraisals-in-thenhs-in-england-innovation-scorecard/to-march-2020/2.estimates-report\#chronic-heart-failure (accessed 10 December 2021).

8. Sankaranarayanan R, Hartshorne-Evans N, Redmond-Lyon S, et al. The impact of COVID-19 on the management of heart failure: a United Kingdom patient questionnaire study. ESC Heart Fail 2021;8:1324-32. https://doi.org/10.1002/ ehf2.13209; PMID: 33463044 